\title{
Consequences of Risk for a Start-Up Business and the Effect of External Setback on Cooperation in a Public Goods Experiment
}

\author{
Nanrui Gong
}

\author{
St. Anne's Belfield School \\ Email:yliu@nufe.edu.cn
}

\begin{abstract}
Every start-up business has to cope with is risky, whether it is originated internally or externally. Even if a business start-up functions without major deficiencies on the inside, external factors such as a market crash, a government fine or an attack from business competitors might seriously undermine the company's profitability. Without the promise of profit, there is a likelihood that the external setback might challenge the cooperation among partners who together founded and invested in the start-up. This paper studies the impact of external setbacks on cooperation in business start-ups by modeling a start-up using a public goods game. Such a public goods game yields results that suggest either the total collapse of cooperation in a start-up group when confronted with an external setback large enough to eliminate profit, the full reorganization of cooperation, or merely partial recovery of cooperation.
\end{abstract}

Keywords: Public Goods, Start-up Firms, Business Risks, Game Theory, Business Cooperation

\section{INTRODUCTION}

Modern business start-ups involve cooperation among founders of the company and collective contribution to the company's good. In a capricious business environment, companies at the stage of small-scale start-ups are relatively vulnerable to setbacks. A start-up might not suffer from internal problems but unexpected outside danger including market fluctuation, government fine and invasive campaigns from competitors might plunge the start-up into severe financial crisis. In consequence, the partners might withdraw their contribution to protect their own interest, stop cooperating and causing the company to collapse. This research investigates the response a start-up would have toward a risk externally generated by modeling the start-up using a public goods game, as well as the reason behind such responses. In further consideration, this paper aims to raise insights on possible directions start-up companies should turn to promote risk aversion abilities in its early stages.

\subsection{Experimental setup}

\subsubsection{The experimental design}

In a standard public goods game, every individual receives the same amount of money the start of every period. Subsequently, they will decide to allocate a part of that money they received to private exchange and the rest to the public exchange as a necessary step of the voluntary contribution mechanism. The amount of money an individual devotes to the public exchange will result in a returned benefit to all group members each with the amount translated from the individual's group contribution through a rate called the marginal per capita return, or MPCR. After contribution decisions were made privately by each individual and all additions to the group exchange returns an equal payoff to each member, the current period is considered finalized and a new period then starts. A public goods game usually contains multiple such periods[1]. In the context of a business start-up, the founding team is freely composed, with each member making voluntary contribution to the company as investment, which resembles the setting of a public goods game. The fact that the entrepreneurship is successful and the company makes profit paid to partners as returns aligns with the logic of a public goods game in which cooperation will result in higher return to the individual. The external setback could be modeled by a sudden decline in marginal per capita return that makes cooperation unprofitable in some periods of the public goods game.

In a standard public goods game with randomly recruited players, average group contribution rate often trends down over period, signaling the general disintegration of cooperation[4]. In a start-up business setting, however, it is generally assumed that invigorated cooperation among founders is critically in place to begin with, that business partners voluntarily collaborate and join funds in a project that they perceive as a common goal. The partners have the desire to collaborate in the first place because of their expectation of bountiful payoff in the case of success. Their desire to collaborate is present even before their cooperation 
starts. Given such impetus, individual partner's involvement in the shared undertaking has the reason to even strengthen with the start-up company maintaining stable growth as time goes on. In order to give adequate attention to this impetus, the population participating in the public goods game needs to be stratified according to their attitudes toward cooperation[5]. Furthermore, composition of players might play as a determining factor in the group showing different responses to a setback. In this respect, a preliminary standard public goods game needs to be conducted to divide participants into categories of unconditional cooperators, conditional cooperators and free riders.

\subsubsection{Unconditional cooperators}

In a standard public goods game, those who tend to make contributions to the group at a relatively constant amount are called unconditional cooperators[2].

\subsubsection{Conditional cooperators}

In a standard public goods game, those whose contribution generally adheres those of others are called conditional cooperators[2].

\subsubsection{Free riders}

In a standard public goods game, those who rarely contribute to the group benefit are called free-riders[2].

\subsubsection{Limitations}

The added element of a random setback in the experiment setting might not perfectly model the setback a startup might face in reality. Real life situations could be a lot more entangled and companies might encounter crisis that is not confined to pure monetary losses.

\subsection{Analysis of possible reactions to the setback mechanism}

\subsubsection{Contribution rate decline for selfish ends, also as a means of self-protection}

Through the experiment one can more directly observe the impact a setback has on cooperation among a group. In the middle of the game, the MPCR will fall significantly at a random period indifferent to any actions of the players and stay depressed for three periods. This additional mechanism mimics a major outside factor that hits hard the normal transaction of the start-up which usually doesn't have the capability to sidestep or to offset the damage because of its size. Traditional game theory establishes the hypothesis that participants in the game are completely rational, or selfinterested. They play along a self-interested principle in maximizing their own gains at any chance[3]. Given this assumption, players in the game should withhold as much as possible their contributions to the group, since significantly lower MPCR means that the ability of group contributions to generate profit is limited. One estimated outcome of this game is that contribution rate which has settled at a high level before the mechanism of setback activates declines rapidly during the three periods when the effect of the setback still remains. After the setback mechanism passes and MPCR returns to original level, contribution dissipates completely or stays at a minimal level for the rest of the game. High contribution will no longer make as much payoff for each individual, making exploiting others' public contribution more appealing. Since it is assumed that players are rational and understand the game fully, they should also recognize that if they keep making large contributions while others gravitate towards free riding, their altruism will be exploited by the latter, which goes against their principle of self-interest. Considering both of these factors, restraining from cooperation would be their dominant strategy. Besides, they should be concerned about the risk of another setback in the unpredictable future which will damage their common and individual interest again if they allocate their resources predominantly to the group exchange the previous round. Therefore, even after the difficult period passes and MPCR goes back to a higher level, there is enough reason to believe that cooperation will not rekindle at all for the rest of the periods. As self-preservation becomes an important imperative, because of the knowledge of an indeterminate risk that might devastate their income again, they will prefer a least possible amount of contribution to the group. Other than for the advantage of free-riding per se, players stop cooperating simply as a conservative strategy in reaction to the fear for another setback. If they don't make group contributions or make little of them, they won't have to lose anything in the case of another setback. Since they don't know when exactly the next setback, if there is one, will descend, they have the reason to be on guard every round and maintain a tight grip of the stakes they hold.

\subsubsection{Difference between conditional cooperators and unconditional cooperators}

Among the players, unconditional cooperators and conditional cooperators stands more true to actual members of a start-up group. Their willingness to cooperate in the game coincides with the voluntary participation in a startup.

\subsubsection{Sorted game with conditional cooperators}

When playing the public goods game with the setback mechanism, it is conjectured that a group made up 
exclusively by conditional cooperators will come out of the setback periods with injured average contribution rate and that their cooperation level will remain low for the rest of the game. As to a normal sorted public goods game without the setback mechanism, previous experiments have shown that conditional cooperators are able to maintain a relatively high contribution level when they're playing with likeminded participants[6]. In a public goods game with the setback mechanism, while their cooperation level remains steady before the setback, a setback will likely cut it down simply because the incentive vanishes, dampening the desire to cooperate. Because of their identity as conditional cooperators, their actions are dependent on others' moves. In the wake of the setback, each of them waits to follow the example of their peers. Theoretically, since cooperation level is relatively low and no one wants to take initiative to expand group contribution, the players will be trapped at a poor contribution rate for the rest of the game. Another reason is that players, when conscious of risk, might favor self-preservation, as mentioned before.

\subsubsection{Sorted game with unconditional cooperators}

In a sorted public goods game with the setback mechanism, unconditional cooperators might manage to bring cooperation among players back up to a high standard, though this potential might not induce a full scale recovery. From past public goods game experiment results, unconditional cooperators achieve the highest average contribution rate when they are playing with others of their type[6]. When the setback element was introduced in the game, cooperation level among unconditional cooperators will most likely fluctuate, since public goods become a more costly and thus more inefficient objective even for those with proactive desire to cooperate. For unconditional cooperators, even if they devote extensive resources to the public exchange, they won't be able to create as much effect under their altruistic imperative, due to the MPCR drop. However, once the influence of the setback period passes, unconditional cooperators will likely revive intense cooperation relatively quickly from the depression. Once MPCR rises, their contribution to the collective benefit can resume as steadily as ever, with the condition that they are unmoved by others' behavior. Nevertheless, cooperation among unconditional cooperators faces the challenge of risk-aversion tendencies. Learning from past experience, players predict that a future setback will again devastate the conversion from group contribution to group earnings, making the public good a more whimsical objective complicated by an outside factor that could not be resolved simply by individual's will or effort. This might cause a loss of interest for achieving a common goal. In calculation of this risk, unconditional cooperators will downsize their contribution. All factors considered, unconditional cooperators will reinforce cooperation after a setback. Their average group contribution rate mostly likely converges to a standard lower than before the setback hit, but cooperation does not falter throughout the entire period.

\subsubsection{Heavy setback might mean incomplete return to the original contribution rate}

In a public goods game with the setback element, the extent to which the average contribution rate will recover might depend on how much the MPCR has declined. There is evidence that higher MPCR in general prompts players to choose a more cooperative mode in[7]. With more dramatic MPCR decline, setbacks are more severe in consequences and inflict a bigger loss for the participant. This should discourage their willingness to bring back cooperation even further. The setback should also leave a more disastrous impression that will aggravate players' worries about the risk of another setback. On the contrary, mild decline in MPCR will likely not produce enough force to overwhelm cooperation.

\section{CONCLUSION}

From previous analysis, in a start-up business running into an external crisis, the cooperation pattern fits the mechanism of a public goods game with stratified participants. When such an experiment is conducted, possible results might be a theoretical perpetual decline of group contribution rate because the self-interested principle naturally favors risk aversion. High group contribution might also sustain after the setback because of a strong faith in the public good. Even when group contribution rate recovers, it might not return to the original level because of predictions about future setbacks. Since a strong belief in the collective achievement is a catalyst for a high recovery of group contribution rate, companies should take special care in fostering a collective spirit and strong morale among partners. Business leaders should also consider setting examples in maintaining high group contribution in the time of crisis in order to counter the damage on group members willingness to keep making contribution. Heavier setbacks might lead to an inadequate recovery of group contributions, so the company might also make priority the task of alleviating the loss for partners imposed by the setback. Due to a lack of experimental data, this study only constructs a revised public goods game that models the case of a startup, explores possible outcomes of a public goods game with an external setback and deduct the reason that cause such behavioral patterns. With data and observations collected from actual experiments, future studies will hopefully provide a more definite answer to the question raised in this study and provide strong enough evidence to validate theories on group reaction to an external setback.

\section{ACKNOWLEDGMENT}

I'd like to express my gratitude for my professor and mentors who offered guidance throughout my writing and worked diligently to helped me shape my article. 


\section{REFERENCES}

[1] D. Davis, Douglas, and Charles A. Holt, "Experimental Economics", Princeton University Press, 1993, pp. 317-333

[2] Fischbacher, Urs, et al. "Are People Conditionally Cooperative? Evidence from a Public Goods Experiment." Institute for Empirical Research in Economics University of Zurich, 2000

[3] "4 Public Goods Experiments and Social Preferences." Economic and Political Weekly, vol. 46, no. 35,2011 , pp. 61-70.

[4] Marks, Melanie, et al. "Cooperation versus Free Riding in a Threshold Public Goods Classroom
Experiment." The Journal of Economic Education, vol. 37, no. 2, 2006, pp. 156-170.

[5] N. Burton-Chellew, Maxwell, et al. "Conditional cooperation and confusion in public-goods experiments." Proceedings of the National Academy of Sciences, 2016

[6] Gächter, Simon, and Christian Thöni. "Social Learning and Voluntary Cooperation Among LikeMinded People." Journal of the European Economic Association, vol. 3, no. 2/3, 2005, pp. 303-314.

[7] Isaac, R. Mark, and James M. Walker. "Group Size Effects in Public Goods Provision: The Voluntary Contributions Mechanism." The Quarterly Journal of Economics, vol. 103, no. 1, 1988, pp. 